\title{
Practical measurement of changes in leg length discrepancy after a myofascial release on the thoracolumbar fascia in patients with acute low back pain. A pilot study.
}

\author{
Andreas Brandl ${ }^{1}$, Christoph Egner ${ }^{1}$, Robert Schleip ${ }^{1,2}$ \\ ${ }^{1}$ DIPLOMA Hochschule, 37242 Bad Sooden-Allendorf, Germany \\ ${ }^{2}$ Conservative and Rehabilitative Orthopedics, Department of Sport and Health Sciences, Technical \\ University of Munich, 80333 Munich, Germany
}

Please cite as: Brandl, A.; Egner, C.; Schleip, R. (2021). Practical measurement of changes in leg length discrepancy after a myofascial release on the thoracolumbar fascia in patients with acute low back pain. A pilot study. SportR $\chi i v$.

https://doi.org/10.31236/osf.io/wvkgr

\section{For correspondence}

E-mail: brandl.andreas@stud.diploma.de

Authors agreement: The authors agree to the sharing of this preprint on SportR $\chi$ iv

\section{Abstract}

Background: Recent work has examined an association between leg length discrepancy (LLD) and acute low back pain (aLBP). Myofascial release (MFR) techniques are thought to be frequently applied in the treatment of aLBP. The purpose of this study was to evaluate a practical measure of LLD and the feasibility of an MFR technique in a randomised controlled trial (RCT).

Methodology: In 12 subjects ( 7 women and 5 men) with aLBP and LLD greater than $3 \mathrm{~mm}$, an MFR technique was performed on the thoracolumbar fascia. At the baseline, after the intervention, and at follow-up, LLD was measured using a cross line laser and finger-to-floor distance (FFD), and pain was measured with a visual analogue scale (VAS). Patients completed a questionnaire after follow-up to assess the acceptability of the study procedure. The therapist evaluated the methods in terms of their feasibility.

Results: LLD measurement and MFR treatment required little time and few resources. Participants agreed to the study procedure with moderate to high acceptance. The LLD decreased by $5.00 \mathrm{~mm}$ after treatment and by $4 \mathrm{~mm}$ at follow-up. The VAS showed a reduction in pain of $17.50 \mathrm{~mm}$ at follow-up but not immediately after treatment.

Conclusion: The measurement of LLD is applicable in daily manual therapy practice, but it cannot be assumed to be a valid method for an RCT. Validated methods such as video raster stereography are therefore recommended. Comprehensive RCTs to study the effects of MFR intervention on leg length are feasible.

Trial registration: German Clinical Trials Register (DRKS00025200) 26.04.2021

Keywords: Thoracolumbar fascia, myofascial release, leg length discrepancy, manual therapy, acute low back pain 


\section{Introduction}

Back pain is a significant health problem in developed countries and is one of the most common reasons for consultations in primary care (Barnes et al., 1997). The prevalence of non-specific low back pain has been reported to be between 30 and $70 \%$ in the age group between 18 and 74 years. The cure rate for acute low back pain (aLBP) is believed to be $90 \%$ within six weeks, while only $2-7 \%$ of patients become chronic (Lampert et al., 2015). However, these data were collected from studies that defined the duration of pain as the time between the doctor's visit and the patient's return to work, rather than studying the actual course of pain (Bundesärztekammer (B ̈̈K) et al., 2017). In a meta-analysis of 11 studies (3118 patients), Itz et al. (2013) found that the rate of spontaneous recovery of aLBP patients in the first three months was only $33 \%$, while $65 \%$ still suffered from low back pain one year after the onset of pain. Pengel et al. (2003) reported recurrence with renewed incapacity to work in $33 \%$ of aLBP cases.

Rannisto et al. (2015) found correlations between functional leg length discrepancies (LLD) and aLBP in workers at a slaughterhouse. Jeevannavar et al. (2018) studied the prevalence of LLD in 245 individuals with non-specific LBP and found that $17 \%$ had different leg lengths. Therefore, they recommended that measurement of LLD should be included in the standard routine examination of LBP patients.

The pelvis is a central part of the kinematic chain between the lower extremities and the spine. An important link in this chain is the sacroiliac joint (SIJ) and its mobility. In a study of 6 human subjects, Vleeming et al. (1992) found motion amplitudes of up to $4.54^{\circ}$ in the SIJ. They hypothesised that the abdominal muscles, the abdominal transverse muscle, the internal oblique muscle, and the external oblique muscle, exert an anteromedially directed force vector on the anterior superior iliac spine (ASIS). This means that both ASIS regions are pulled toward each other, which increases the pressure on the articular surfaces of the SIJs. However, this biomechanical model only works if a posterior force prevents the two iliac bones from sliding apart in the posterior superior iliac spine (PSIS) region (Willard et al., 2012). In this regard, Willard et al. (2012) attribute an important role to the thoracolumbar fascia (TLF), along with the multifidus muscle and sacral ligaments. The TLF increases in cross-section, especially in the region caudal to L5 and the os sacrum, and unites with fibres of the sacroiliac ligaments. Other important structures in this power-lock system are the muscles that connect to the sacrotuberous ligament, such as the gluteus maximus muscle, and, in some individuals, the biceps femoris muscle (Panjabi, 2006).

Myofascial release (MFR) techniques are frequently used in manual medicine with the aims of restoring the optimal length of myofascial tissue structures, improving their function, and reducing their pain (Ajimsha et al., 2015). Some studies have investigated the efficacy in this regard with conflicting results (Ajimsha and Shenoy, 2019; Chen et al., 2016; Laimi et al., 2017; Wong et al., 2017). Ajimsha et al. (2015), in a systematic review examining 19 randomised controlled trials of MFR, noted that research on the efficacy of MFR is still in its infancy. To the authors' knowledge, no other work has examined the relationship between an MFR at the TLF, pelvic stability, and LLD. This is the first study that has been conducted to evaluate such a relationship.

\section{Aims}

It is important that an MFR intervention is shown to be feasible and accepted by subjects before full-scale randomised controlled trials (RCTs) are conducted. Therefore, the primary objectives of this study were to investigate this and the feasibility of measuring LLD. The secondary objectives were to collect measurement time point (MT) data and to estimate the treatment effect in order to calculate sample sizes for subsequent RCTs.

\section{Methods}

\section{Study design overview}

A pilot study was conducted with an open pre-post-test single-group design. Measurements were taken before and after the intervention plus one day later, following the SPIRIT guidelines (Chan et al., 2013). The study protocol was registered with the German Registry for Clinical Trials (DRKS00025200) on 26/04/2021. The study was reviewed and approved by the Ethics Committee of the Osteopathic Research Institute in Hamburg, Germany (No. 018-05), was conducted in accordance with the Declaration of Helsinki (World Medical Association, 2013), and informed consent was obtained from all participants.

\section{Setting and participants}

The study was conducted in an osteopathic practice in southern Germany. Subjects between 18 and 50 years of age with acute or subacute low back pain who achieved a minimum score of 20 on the Oswestry Disability Questionnaire (German version) (ODQ-D) (Gaul et al., 2008) and with pain duration of less than 13 weeks (Bundesärztekammer (B ̈̈K) et al., 2017) were recruited using a direct approach, a notice board, and distribution of information material in the practice or to acquaintances.

\section{Inclusion criteria}

Inclusion criteria: acute or subacute lumbar back pain, as defined by the European guidelines for the management of acute low back pain (van Tulder et al., 2006); minimum 
score of 20 on the ODQ-D; less than 13 weeks pain duration; female or male subjects aged 18 to 50 years; prone position for 15 minutes must be pain-free for the subjects.

\section{Exclusion criteria}

Exclusion criteria: generally valid contraindications to physiotherapeutic and manual treatments of the lumbar spine and pelvis; rheumatic diseases; scoliotic changes of the lumbar spine; consumption of medications that affect blood coagulation; consumption of muscle relaxants; expression of skin changes (e.g. neurodermatitis, psoriasis, urticaria, decubitus ulcers); surgery or scars in the lumbar region between Th12 and S1.

\section{Intervention}

The subjects were first screened for eligibility by the principal investigator $(\mathrm{AB})$, who then performed the baseline measurements. The subjects received the MFR treatment from a therapist with more than 10 years' professional experience in manual therapy. This was followed by measurements after the intervention and a follow-up one day later.

The participants received an intervention as described by Chila and O'Connell (2010). In this intervention, the subject is in a prone position with the arms at the sides of the body and the legs parallel to each other. The head is in a neutral position and the face is in a recess in the head section of the therapy table. The patient is sufficiently undressed to access the TLF between Th12 and S1. The therapist stands contralateral to the side to be treated at the level of the subject's iliac crest (Figure 1). The therapist's cranial hand, which serves as the palpating hand, is positioned dorsally immediately adjacent to the lumbar spine, extensively touching the TLF at the level of L1 to L4. The caudal hand duplicates the palpating hand and initiates a direct stretch of the fascia laterally to a palpable tissue resistance.

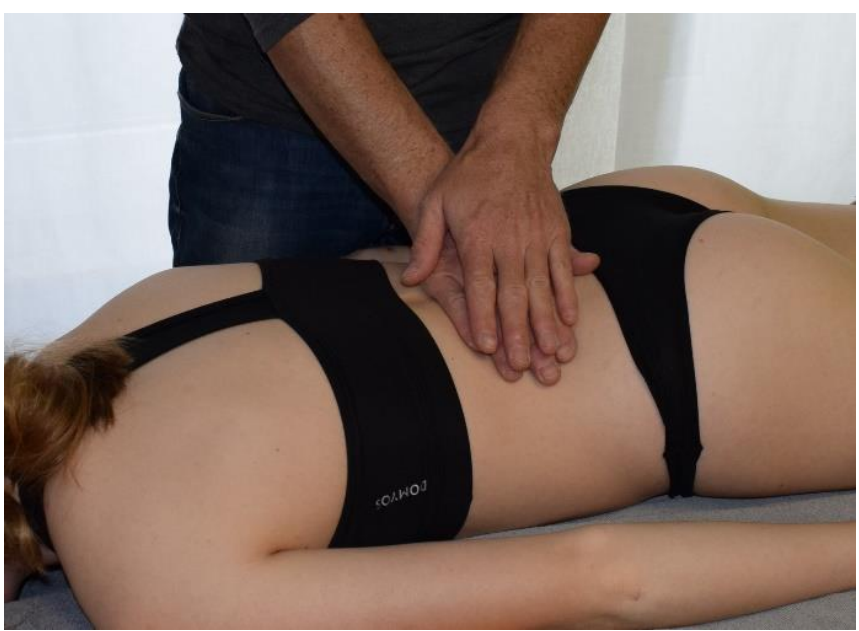

Figure 1. MFR treatment
The therapist follows the viscoelastic creep effect of the myofascial tissue to initiate further stretching of the TLF (Willard et al., 2012). The traction torque applied to the tissue is moderate, ranging from 25 to $35 \mathrm{Nm}$ and acting tangentially laterally toward the abdominal muscles. The standard force applied during an MFR treatment was previously trained by the therapist using a phantom pad placed over a highly sensitive force plate. The training was considered sufficient when the blinded practitioner was able to perform 30 stroke applications on the force plate, all of which were within this force range, with no outliers. This training was repeated once per day during data collection. The duration of the entire technique is 60 to 90 seconds. However, the crucial factor for the effect is not the amount of time over which the technique is practised, but the occurrence of the MFR effect. Ajimsha et al. (2015) define the MFR effect as the restoration of the optimal length of myofascial tissue structures, their functional improvement, and the reduction of pain in them.

\section{Primary outcome measurements}

LLD was measured with a cross line laser. Subjects' long hair was tied with a hair tie or similar if necessary. The participants then stood barefoot and unclothed except for their underwear with their back to the measuring device.

The sacroiliac dimples were marked with black dots to determine the height of the PSIS. A horizontal line was then projected through the left dot (Figure 2). The LLD is measured as the difference between

the laser line and the right PSIS (Bruzek,

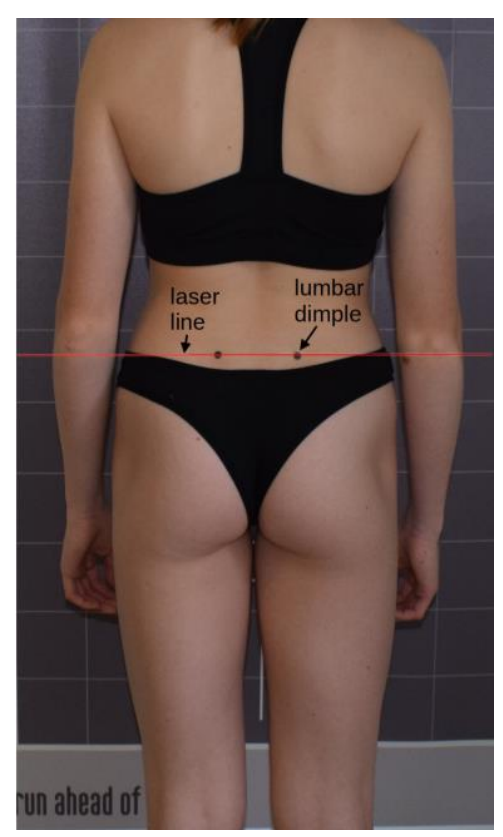

Figure 2. Cross-line laser 2006). The measurement accuracy of the laser projection system is $0.5 \mathrm{~mm} / \mathrm{m}$. The minimum PSIS change that can be detected by manual palpation has been reported to be between 3 and $5 \mathrm{~mm}$, depending on the individual skill of the examiner (Cooperstein and Hickey, 2016; Lavazza et al., 2018; O'Haire and Gibbons, 2000). This assessment was used as primary outcome parameter, based on practicality for the evaluator. The time required for a complete measurement was also recorded.

As another primary outcome parameter, the subjects' acceptance of the intervention and the measurement procedure 
was evaluated by a post-treatment survey with a five-point Likert scale.

\section{Secondary outcome measurements}

The LLD was determined using cross line laser measurement. In addition, pain was assessed using a visual analogue scale (VAS).

To measure the finger-to-floor distance (FFD), the patients stood with bare feet hip-width apart on a platform to which a millimetre measure was attached. The patients were asked to place their right palm on the back of their left hand and to bend their hips as far as possible with their fingers, arms, and knees extended. The examiner then read the maximum flexion value achieved on the scale. A positive value marked a final position above the stance plane, with a negative value indicating a final position below the stance plane. Ekedahl et al. (2012) considered $45 \mathrm{~mm}$ to be the minimally detectable change (MDC).

\section{Statistical analysis}

Participant acceptability was assessed using the median, $25 \%$ quartile (Q1), and $75 \%$ quartile (Q3). For the secondary outcome parameters, the standard deviation (SD), standard error of the mean (SEM), mean, minimum (min) and maximum (max) values, median, Q1, and Q3 were obtained for each MT. Missing data from the follow-up measurement (missing completely at random or missing at random) were excluded pairwise. The sample size for future RCTs with two groups and three MTs was calculated with an $\alpha$-error of 0.05 and a $1-\beta$ error of 0.95 . The effect size was estimated using the mean differences between the baseline measurements and follow-ups from this pilot study and from a previous paper by Barnes et al. (1997). The effects from an RCT conducted by Wong et al. (2017) were also accounted for by the $f$ value from an analysis of variance (Lenhard and Lenhard, 2016).

LibreOffice Calc version 6.4.7.2 (Mozilla Public License v2.0) was used for descriptive statistics. Inferential statistics were carried out with the software R, version 3.4.1 (R Foundation for Statistical Computing, Vienna, Austria). Statistical power was calculated with GPower (C) 1992-2014 Franz Faul, University of Kiel, available at https://www.psychologie.hhu.de/arbeitsgruppen/allgemeinepsychologie-und-arbeitspsychologie/gpower, accessed on 09 September 2021).

\section{Results}

The anthropometric data and baseline characteristics are shown in Table 1.
Table 1 Baseline characteristics

\begin{tabular}{lllll}
\hline Variable & mean $\pm \mathrm{SD}$ & median $(\mathrm{Q} 1-\mathrm{Q} 3)$ & $\min -\max$ & $\mathrm{n}$ \\
\hline Age $($ years $)$ & $40,6 \pm 10,8$ & $43,4(41,2-47,1)$ & $18,3-50,1$ & 12 \\
Gender $(\mathrm{m} / \mathrm{f})$ & $5 / 7$ & & & 12 \\
Height $(\mathrm{m})$ & $1,74 \pm 0,1$ & $1,73(1,68-1,82)$ & $1,60-1,90$ & 12 \\
Weight $(\mathrm{kg})$ & $77,2 \pm 19,9$ & $73,6(61,9-90,5)$ & $47,0-119,0$ & 12 \\
BMI $\left(\mathrm{kg} / \mathrm{m}^{2}\right)$ & $25,3 \pm 5,4$ & $23,6(22,3-27,8)$ & $17,9-35,9$ & 12 \\
LLD $(\mathrm{mm})$ & $6,1 \pm 1,2$ & $6,00(5,0-7,0)$ & $4-8$ & 12 \\
VAS $(0-100)$ & $28,5 \pm 22,7$ & $21,5(14,5-47,8)$ & $0,0-75,0$ & 12 \\
ODQ-D (0-100) & $25,2 \pm 6,1$ & $23,0(20,0-28,5)$ & $20,0-36,0$ & 12 \\
\hline
\end{tabular}

\section{Primary objectives}

Of the 14 subjects screened, 12 met the eligibility criteria and received the intervention (Figure 3); 14\% did not meet the eligibility criteria. Three participants dropped out of the study during follow-up. The principal investigator described the time and effort required to perform the cross line laser measurement as 'minimal'.

The results of the follow-up survey of the patients' acceptance on the five-point Likert scale are shown in Table 2. The participants rated the individual measurements and the manageability of the two examination appointments.

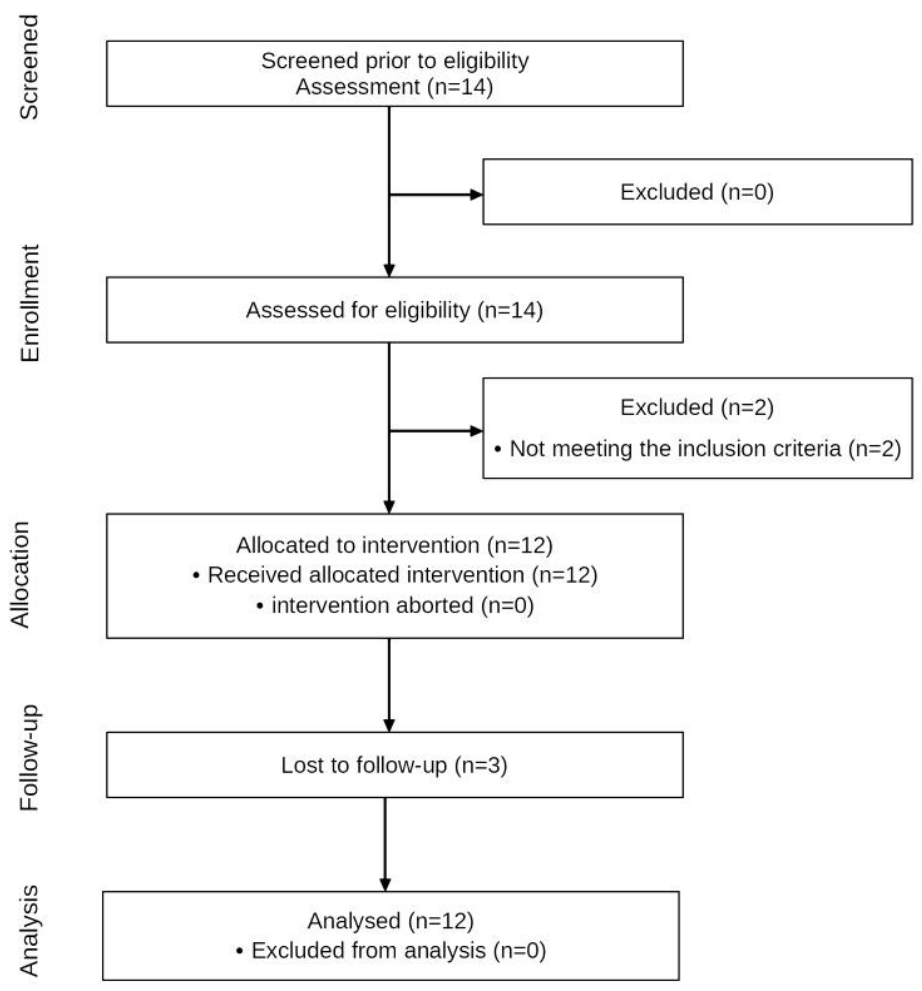

Figure 3. Flow chart of the study 
Table 2 Follow-up survey of participant acceptance

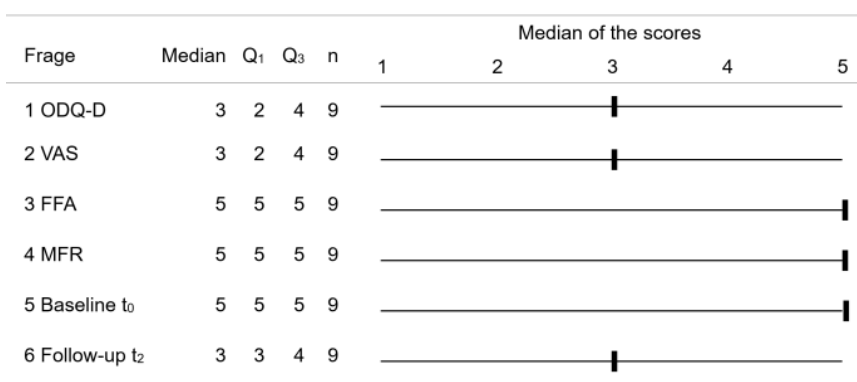

\section{Secondary objectives}

The changes in LLD between baseline $\left(\mathrm{t}_{0}\right)$, post-treatment measurement $\left(t_{1}\right)$ and follow-up $\left(t_{2}\right)$ are shown in Figure 4 and Table 3.

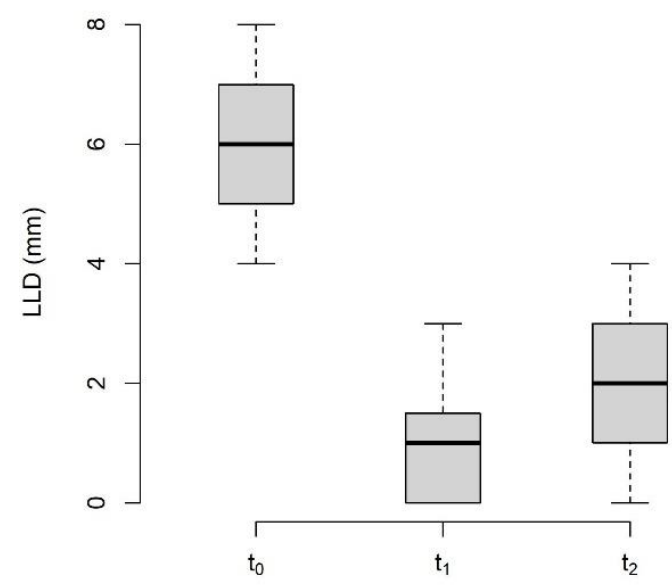

Figure 4. Leg length discrepancy changes

Table 3 Descriptive statistics LLD (values in $\mathrm{mm}$ )

\begin{tabular}{llllllllll}
\hline MT & mean & SD & SEM & median & Q1 & Q3 & $\min$ & $\max$ & $\mathrm{n}$ \\
\hline $\mathrm{t}_{0}$ & 6,08 & 1,24 & 0,36 & 6,00 & 5,00 & 7,00 & 4 & 8 & 12 \\
$\mathrm{t}_{1}$ & 0,92 & 1,00 & 0,29 & 1,00 & 0,00 & 1,25 & 0 & 3 & 12 \\
$\mathrm{t}_{2}$ & 2,00 & 1,50 & 0,92 & 2,00 & 1,00 & 3,00 & 0 & 4 & 9 \\
\hline
\end{tabular}

The changes in FFD between baseline $\left(\mathrm{t}_{0}\right)$, post-treatment measurement $\left(\mathrm{t}_{1}\right)$ and follow-up $\left(\mathrm{t}_{2}\right)$ are shown in Figure 5 and Table 4.

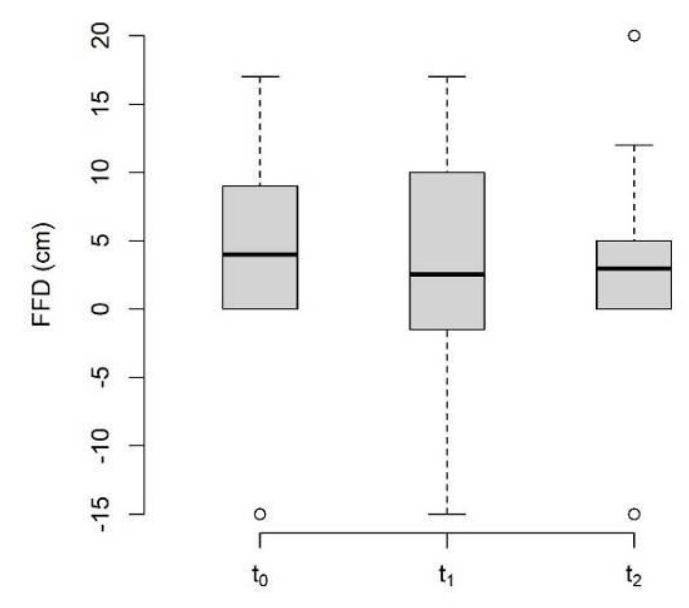

Figure 5. Finger-to-floor distance changes

Table 4 Descriptive statistics FFD (values in $\mathrm{cm}$ )

\begin{tabular}{llllllllll}
\hline MT & mean & SD & SEM & median & Q1 & Q3 & $\min$ & $\max$ & $\mathrm{n}$ \\
\hline $\mathrm{t}_{0}$ & 4,00 & 8,06 & 2,33 & 4,00 & 0,00 & 7,50 & -15 & 17 & 12 \\
$\mathrm{t}_{1}$ & 2,83 & 9,28 & 2,68 & 2,50 & $-3,50$ & 9,00 & -15 & 17 & 12 \\
$\mathrm{t}_{2}$ & 2,11 & 9,32 & 2,83 & 3,00 & 0,00 & 5,00 & -15 & 20 & 9 \\
\hline
\end{tabular}

The changes in VAS between baseline $\left(\mathrm{t}_{0}\right)$, post-treatment measurement $\left(\mathrm{t}_{1}\right)$ and follow-up $\left(\mathrm{t}_{2}\right)$ are shown in Figure 6 and Table 5.

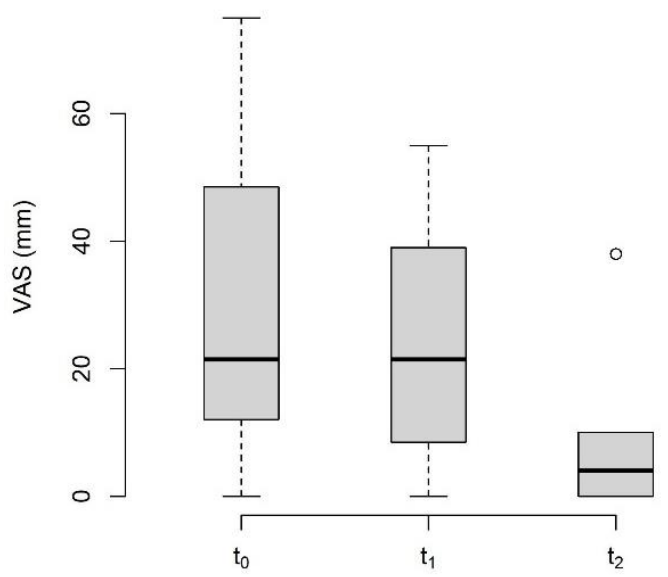

Figure 6. Visual analogue scale changes

Table 5 Descriptive statistics VAS (values in $\mathrm{mm}$ )

\begin{tabular}{llllllllll}
\hline MT & mean & SD & SEM & median & Q1 & Q3 & $\min$ & $\max$ & $\mathrm{n}$ \\
\hline $\mathrm{t}_{0}$ & 28,50 & 22,6 & 6,55 & 21,50 & 14,50 & 47,75 & 0 & 75 & 12 \\
$\mathrm{t}_{1}$ & 24,25 & 18,3 & 5,30 & 21,50 & 10,25 & 36,00 & 0 & 55 & 12 \\
$\mathrm{t}_{2}$ & 7,78 & 12,0 & 3,48 & 4,00 & 0,00 & 10,00 & 0 & 38 & 9 \\
\hline
\end{tabular}

The LLD effect size between the baseline measurement and follow-up was $\mathrm{f}=0.75$. The effect size of the study by Barnes et al. was $f=0.90$ and that of the RCT by Wong et al. 
was $\mathrm{f}=0.57$ (Barnes et al., 1997; Wong et al., 2017). Based on the considerable uncertainty regarding the assumed intervention effects of MFR, we followed the recommendations of Ajimsha and Shenoy (2019) and assumed a medium effect size $(f=0.25)$. Power analysis yielded a total sample size of 44 , corresponding to a power of $95 \%$ and a significance level of $\alpha=0.05$. For future comprehensive RCTs, it is recommended to allow for $10 \%$ loss to follow-up. A sample size of 50 participants (25 in each group) would support this.

\section{Discussion}

\section{Primary objectives}

The results of this study show that RCTs with an MFR treatment group are feasible, but some modifications are needed. The cross line laser method of measuring LLD achieved the MDC at the post-intervention MT in 7 of 12 cases and at follow-up in only 2 of 9 cases. Despite the large difference in mean and the low SD, it cannot be considered a valid method for use under the conditions of an RCT. The reliability is too dependent on the individual experience of the examiner palpating the PSIS for cross line laser detection. Cooperstein and Hickey (2016) found in a systematic review that none of the included studies achieved 'moderate' intraor inter-examiner reliability $(\kappa<40)$. However, reliability increases sharply with investigator experience. Lavazza et al. (2018) found that only $50 \%$ of osteopathic students were able to correctly palpate the PSIS. In contrast, professionals with more than 4 years of practical experience achieved a detection rate of $84 \%$. Thus, the method can be recommended for daily assessment in an osteopathic or manual therapy practice for therapists with expertise. In this case, it is an uncomplicated, easy-to-use tool for monitoring treatment effects.

The volunteers gave an agreement score of only 3 out of 5 points for the VAS and ODQ-D in the follow-up survey. It is known that many patients fear that grading pain on a scale does not do justice to their individual complex situation (Karner, 2012). This may influence the acceptance of pain measurement methods. At follow-up, 3 subjects dropped out. The manageability of a second measurement session one day after the first was rated only 3 out of 5 . This needs to be addressed in future RCTs. The LLD, MFR, and manageability of the first appointment were scored as the maximum of 5 points. It can therefore be assumed that the design and procedure of the pilot study, with the exception of the aforementioned items, are feasible and practicable for future RCTs.

\section{Secondary objectives}

Interpretation of changes in the LLD after treatment is difficult because of the limitations mentioned above. However, 7 participants exceeded the MDC immediately after the intervention. Therefore, the mechanisms that might cause this should be discussed. When a unilaterally stiffened TLF is released, the iliac bones can rotate toward symmetry relative to the sacral bone. The MFR investigated in this pilot study could cause an effect as described by Chen et al. (2016) for these structures. The tissue stiffness of the middle layer of the TLF, with its connections to the transverse abdominal muscle, could also decrease significantly as a result, initiating an anterior sliding of the pelvic myofascial corset system toward the umbilicus. This would move the ilia toward each other with an anteromedial force vector and allow diarthrotic joint behaviour of the SIJ (Vleeming et al., 1992). The ilium rotates anteriorly and the LLD decreases compared to the baseline measurement. An alternative explanation could be altered neuromuscular behaviour of the erector spinae muscles, causing functional scoliosis and LLD (Schleip et al., 2019; Stecco et al., 2013). Proprioception may be affected by adhesions between soft tissue layers, especially the muscle spindles, which could be unilaterally blocked and alter the behaviour of the erector spinae muscles (Stecco et al., 2013). MFR intervention can influence these mechanisms such that the soft tissue layers regain their ability to glide on each other and unblock the muscle spindles.

The FFD was reduced by $1.17 \mathrm{~cm}$, but this value did not reach the MDC of $4.5 \mathrm{~cm}$ (Ekedahl et al., 2012). The differences in mean values for each MT were marginal, with a large SD. Ekedahl et al. (2012) claimed that the FFD had good validity in patients with aLBP. The authors of the study examined a group of subjects over a period of several months and were able to describe the FFD as a good predictor of self-documented level of disability. Nevertheless, based on the results of this pilot study, FFD appears to be inappropriate for measuring the short-term effects of MFR on TLF. This is in line with the results of a study by Köck (2015), who also investigated the effects of MFR on the FFD and did not find any clinically relevant short-term changes.

The subjects were unable to document a change in subjective pain sensation after MFR using the VAS. Nonetheless, data analysis showed changes both from the baseline measurement to follow-up $(-20.72 \mathrm{~mm})$ and between postintervention measurement and follow-up $(-16.47 \mathrm{~mm})$. The MDC was exceeded by 6 of the 9 subjects (Schomacher, 2008). These 6 subjects were all new to the manual therapy practice where the study was conducted and it can be assumed that they received follow-up treatment there. An effect based on the expectation of symptom improvement due to upcoming therapy can therefore not be excluded.

A robust estimate of the intervention effect is needed to calculate the sample size for a comprehensive RCT. To the best of the authors' knowledge, this was the first study to examine the association between a single MFR treatment on the TLF and associated changes in LLD. Sample size calculations should not be based solely on the effect of a single- 
arm pilot study with a small sample size. For this reason, previous MFR studies were also included in the estimation of effect size. Unfortunately, limitations (e.g. small sample sizes, incongruent interventions, methodological limitations) cannot be completely ruled out, so the recommendation of Ajimsha and Shenoy (2019) was followed and a medium effect size was assumed. The power analysis then yielded a total sample size of 50, which can be recruited during the daily routine of a manual therapy practice.

\section{Recommendations for a future comprehensive RCT}

1. Cross-line laser measurement with manual palpation of the LLD should be replaced by validated methods, e.g. video raster stereography (Degenhardt et al., 2020).

2. When setting the follow-up appointment, the participants' wishes should be taken into account.

3. The FFD seems unsuitable for measuring short-term effects of an MFR on the TLF.

4. The results of the VAS and ODQ-D measurements should be evaluated with caution and with respect to the individual circumstances of the volunteers.

5. The sample size for a two-arm RCT should only consider a medium effect and include 25 participants per group.

\section{Limitations of the study}

This pilot study had several strengths, particularly participant acceptance of the MFR intervention and measurement methods. The potential limitations of this study have been described above. It is important not to interpret the results as evidence of the effectiveness of MFR. There was only one intervention group, which was not blinded. There was no sham control, and the therapist could not be blinded as to which intervention he gave to the subjects. Aside from whether MFR has an effect, it will also be interesting to see how it compares to other techniques and to normal osteopathic or manual therapy. This could be the theme of subsequent RCTs, which may include one or several additional intervention groups.

The limitations should be seen in light of the fact that the primary aim of this pilot study was to prepare and make recommendations for subsequent RCTs.

\section{Conclusion}

The results of this pilot study support the feasibility of subsequent RCTs to investigate the effect of fascia treatment on functional leg length. Cross line laser measurement with manual palpation does not appear to be sufficiently objectifiable and validatable under the conditions of an RCT to provide usable results regarding functional leg length. Nevertheless, it may be a useful tool for monitoring treatment effects in daily practice. The subjects were positive about the meth- ods in the follow-up survey, with the exception of the pain measurement and follow-up one day after the first appointment.

The FFD measurement should not be included in future short-term intervention studies because it cannot determine the short-term effects of MFR. The VAS score should be considered with caution, taking into account the individual circumstances of the participants and the setting in which the study is conducted.

Comprehensive studies would need to include at least 50 volunteers for an intervention and a control group.

The results of this feasibility study suggest that comprehensive RCTs with an appropriate number of participants for statistical significance are promising and feasible within the setting of a manual therapy practice.

\section{Abbreviations}

aLBP: Acute low back pain; ASIS: Anterior superior iliac spine; FFD: Finger-floor distance; LLD: Leg length discrepancy; max: maximum; min: minimum; MDC: Minimal detectable changes; MFR: Myofascial release; MT: Measurement time point; n: number; Q1: $25 \%$ quartile; Q3: $75 \%$ quartile; ODQ-D: Oswestry Disability Questionnaire (German version); PSIS: Posterior superior iliac spine; RCT: randomized controlled trial; SD: standard deviation; SEM: standard error of the mean; TLF: Thoracolumbar fascia; VAS: Visual analogue scale

\section{Discloser}

The authors have no personal financial or institutional interest in any of the materials or devices described in this article.

\section{Funding}

This research did not receive any specific grant from funding agencies in the public, commercial, or not-for-profit sectors.

\section{References}

Ajimsha, M.S., Al-Mudahka, N.R., Al-Madzhar, J.A., 2015. Effectiveness of myofascial release: systematic review of randomized controlled trials. J Bodyw Mov Ther 19, 102-112. https://doi.org/10.1016/j.jbmt.2014.06.001

Ajimsha, M.S., Shenoy, P.D., 2019. Improving the quality of myofascial release research. A critical appraisal of systematic reviews. J Bodyw Mov Ther. https://doi.org/10.1016/j.jbmt.2019.03.011

Barnes, M.F., Gronlund, R.T., Little, M.F., Personius, W.J., 1997. Efficacy study of the effect of a myofascial release treatment technique on obtaining pelvic symmetry. J Bodyw Mov Ther 1, 289-296. https://doi.org/10.1016/S1360-8592(97)80064-2

Bruzek, R., 2006. Messgeräte, in: Bruzek, R. (Ed.), Leitfaden Gelenkmessung. Elsevier, München, pp. 27-53.

Bundesärztekammer (BÄK), Kassenärztliche Bundesvereinigung (KBV), Arbeitsgemeinschaft der Wissenschaftlichen Medizinischen Fachgesellschaften (AWMF), 2017. Nationale Versorgungsleitlinie Nicht-spezifischer Kreuzschmerz - Langfassung, 2. Auflage. ed. Bundesärztekammer; Kassenärztliche Bundesvereinigung; Arbeitsgemeinschaft der Wissenschaftlichen Medizinischen Fachgesellschaften. https://doi.org/10.6101/AZQ/000377 
Chan, A.-W., Tetzlaff, J.M., Gøtzsche, P.C., Altman, D.G., Mann, H., Berlin, J.A., Dickersin, K., Hróbjartsson, A., Schulz, K.F., Parulekar, W.R., 2013. SPIRIT 2013 explanation and elaboration: guidance for protocols of clinical trials. BMJ 346. https://doi.org/10.1136/bmj.e7586

Chen, Y.-H., Chai, H.-M., Shau, Y.-W., Wang, C.-L., Wang, S.-F., 2016. Increased sliding of transverse abdominis during contraction after myofascial release in patients with chronic low back pain. Man Ther 23, 69-75. https://doi.org/10.1016/j.math.2015.10.004

Chila, A.G., O’Connell, J.A. (Eds.), 2010. Foundations of Osteopathic Medicine, 3. Aufl. ed. Lippincott Williams \& Wilkins, London.

Cooperstein, R., Hickey, M., 2016. The reliability of palpating the posterior superior iliac spine: a systematic review. J Can Chiropr Assoc 60, 36-46. https://doi.org/PMID: 27069265

Degenhardt, B.F., Starks, Z., Bhatia, S., 2020. Reliability of the DIERS Formetric 4D Spine Shape Parameters in Adults without Postural Deformities. BioMed Res Int 2020. https://doi.org/10.1155/2020/1796247

Ekedahl, H., Jönsson, B., Frobell, R.B., 2012. Fingertip-to-floor test and straight leg raising test: validity, responsiveness, and predictive value in patients with acute/subacute low back pain. Arch Phys Med Rehabil 93, 2210-2215. https://doi.org/10.1016/j.apmr.2012.04.020

Gaul, C., Mette, E., Schmidt, T., Grond, S., 2008. ODQ - Oswestry Low Back Pain Disability Questionnaire - deutsche Fassung. BioMed Res Int 2008, 51-58. https://doi.org/10.23668/PSYCHARCHIVES.390

Itz, C.J., Geurts, J.W., van Kleef, M., Nelemans, P., 2013. Clinical course of non-specific low back pain: a systematic review of prospective cohort studies set in primary care. Eur J Pain 17, 5-15. https://doi.org/10.1002/j.1532-2149.2012.00170.x

Jeevannavar, J.S., Ganesh, G.A., Jeevannavar, S.S., 2018. Prevalence of Leg Length Discrepancy in Persons with Non-Specific Low Back Pain. Indian J Physiother Occup Ther 12, 58. https://doi.org/10.5958/09735674.2018.00038.2

Karner, J.J., 2012. Die Abbildung chronischer Schmerzen anhand von validierten Fragebögen - Eine qualitative Studie bei älteren Patienten mit chronischen Schmerzen der Halswirbelsäule (Dissertation). Medizinischen Fakultät Charité - Universitätsmedizin Berlin (Dissertation), Berlin.

Köck, J., 2015. Änderung der Viskoelastizität im Bereich des lumbalen Weichteilgewebes durch eine osteopathische Technik an der Fascia thoracolumbalis und deren Einfluss auf die Rumpfflexion im Stand. Dresden International University (Masterthesis), Dresden.

Laimi, K., Mäkilä, A., Bärlund, E., Katajapuu, N., Oksanen, A., Seikkula, V., Karppinen, J., Saltychev, M., 2017. Effectiveness of myofascial release in treatment of chronic musculoskeletal pain: a systematic review. Clin Rehabil 1. https://doi.org/10.1177/0269215517732820

Lampert, T., Prütz, F., Seeling, S., Starker, A., Kroll, L.E., Rommel, A., Ryl, L., Ziese, T., 2015. Gesundheit in Deutschland: Gesundheitsberichterstattung des Bundes, gemeinsam getragen von RKI und Destatis. Robert Koch-Institut.

Lavazza, C., Milano, V., Abenavoli, A., Maggiani, A., 2018. How type and number of training sessions influence the reliability of palpation. $\mathrm{J}$ Bodyw Mov Ther 22, 396-401. https://doi.org/10.1016/j.jbmt.2017.07.012

Lenhard, W., Lenhard, A., 2016. Berechnung von Effektstärken. Dettelbach: Psychometrica. https://doi.org/10.13140/RG.2.2.17823.92329
O'Haire, C., Gibbons, P., 2000. Inter-examiner and intra-examiner agreement for assessing sacroiliac anatomical landmarks using palpation and observation: pilot study. Man Ther 5, 13-20. https://doi.org/10.1054/math.1999.0203

Panjabi, M.M., 2006. A hypothesis of chronic back pain: ligament subfailure injuries lead to muscle control dysfunction. Eur Spine J 15, 668-676. https://doi.org/10.1007/s00586-005-0925-3

Pengel, L.H.M., Herbert, R.D., Maher, C.G., Refshauge, K.M., 2003. Acute low back pain: systematic review of its prognosis. BMJ 327, 323. https://doi.org/10.1136/bmj.327.7410.323

Rannisto, S., Okuloff, A., Uitti, J., Paananen, M., Rannisto, P.-H., Malmivaara, A., Karppinen, J., 2015. Leg-length discrepancy is associated with low back pain among those who must stand while working. BMC Musculoskelet Disord 16, 110. https://doi.org/10.1186/s12891-015-0571-9

Schleip, R., Gabbiani, G., Wilke, J., Naylor, I., Hinz, B., Zorn, A., Jäger, H., Breul, R., Schreiner, S., Klingler, W., 2019. Fascia is able to actively contract and may thereby influence musculoskeletal dynamics: a histochemical and mechanographic investigation. Front Physiol 10, 336. https://doi.org/10.3389/fphys.2019.00336

Schomacher, J., 2008. Gütekriterien der visuellen Analogskala zur Schmerzbewertung. Physioscience 4, 125-133. https://doi.org/10.1055/s2008-1027685

Stecco, A., Gesi, M., Stecco, C., Stern, R., 2013. Fascial components of the myofascial pain syndrome. Curr Pain Headache Rep 17, 352. https://doi.org/10.1007/s11916-013-0352-9

van Tulder, M., Becker, A., Bekkering, T., Breen, A., Gil del Real, M.T., Hutchinson, A., Koes, B., Laerum, E., Malmivaara, A., 2006. European guidelines for the management of acute nonspecific low back pain in primary care. Eur Spine J 15, s169-s191. https://doi.org/10.1007/s00586006-1071-2

Vleeming, A., Buyruk, H.M., Stoeckart, R., Karamursel, S., Snijders, C.J., 1992. An integrated therapy for peripartum pelvic instability: a study of the biomechanical effects of pelvic belts. Am. J. Obstet. Gynecol. 166, 1243-1247. https://doi.org/10.1016/s0002-9378(11)90615-2

Vleeming, Andry, Buyruk, H.M., Stoeckart, R., Karamursel, S., Snijders, C.J., 1992. An integrated therapy for peripartum pelvic instability: A study of the biomechanical effects of pelvic belts. American Journal of Obstetrics and Gynecology 166, 1243-1247. https://doi.org/10.1016/S0002-9378(11)90615-2

Willard, F.H., Vleeming, A., Schuenke, M.D., Danneels, L., Schleip, R., 2012. The thoracolumbar fascia: anatomy, function and clinical considerations. J. Anat. 221, 507-536. https://doi.org/10.1111/j.14697580.2012.01511.x

Wong, K.-K., Chai, H.-M., Chen, Y.-J., Wang, C.-L., Shau, Y.-W., Wang, S.F., 2017. Mechanical deformation of posterior thoracolumbar fascia after myofascial release in healthy men: A study of dynamic ultrasound imaging. Musculoskelet Sci Pract 27, 124-130. https://doi.org/10.1016/j.math.2016.10.011

World Medical Association., 2013. World Medical Association Declaration of Helsinki: ethical principles for medical research involving human subjects. JAMA 310, 2191-4. https://doi.org/10.1001/jama.2013.281053 